\title{
Chronopharmacological Analysis of Antidepressant Activity of a Dual-Action Serotonin Noradrenaline Reuptake Inhibitor (SNRI), Milnacipran, in Rats
}

\author{
Hiroshi Kawai, ${ }^{* a}$ Megumi Machida, ${ }^{b}$ Takuya Ishibashi, ${ }^{b}$ Naomi Kudo, ${ }^{a}$ Yoichi Kawashima, ${ }^{a}$ and \\ Atsushi Mitsumoto ${ }^{b}$ \\ ${ }^{a}$ Faculty of Pharmacy and Pharmaceutical Sciences, Josai University; 1-1 Keyakidai, Sakado, Saitama 350-0295, \\ Japan: and ${ }^{b}$ Faculty of Pharmaceutical Sciences, Josai International University; 1 Gumyo, Togane, Chiba 283-8555, \\ Japan.
}

Received September 10, 2017; accepted November 22, 2017

\begin{abstract}
Biological rhythms are thought to be related to the pathogenesis and therapy of various diseases including depression. Here we investigated the influence of circadian rhythms on the antidepressant activity of the dual-action serotonin-noradrenaline reuptake inhibitor (SNRI) milnacipran. Rats administered milnacipran in the morning (8:00 a.m.; zeitgeber time [ZT]1) or in the evening (8:00 p.m.; ZT13) were analyzed in a forced swim test (FST). At ZT1, the rats' immobility was reduced and the swimming was increased, whereas at ZT13, their climbing was increased. These results suggest that the serotonergic and noradrenergic systems are preferentially affected at $\mathrm{ZT1}$ and $\mathrm{ZT13}$, respectively by milnacipran. We analyzed the plasma and brain levels of milnacipran after administration, and there were no differences between ZT1 and ZT13. The circadian rhythm of monoamine neurotransmitters was analyzed in several brain regions. The serotonin turnover showed rhythms with a peak during ZT18-ZT22 in hippocampus. The noradrenaline turnover showed rhythms with a peak during ZT22-ZT2. There was a difference of approx. $4 \mathrm{~h}$ between the serotonergic and noradrenergic systems. This time difference might be one of the factors that affect the action of milnacipran and contribute to the dosing time-dependent behavioral pattern in the FST.
\end{abstract}

Key words circadian rhythm; antidepressant; forced swim test; milnacipran; serotonin; noradrenaline

Many physiological functions have a rhythm with a period of $24 \mathrm{~h}$. In mammals, circadian clock systems govern almost all organs, and they affect the pathophysiology of various diseases such as cancer, cardiovascular diseases, and psychiatric diseases. ${ }^{1-4)}$ The circadian clock systems also affect the efficacy of medication by influencing the pharmacokinetics and/or pharmacodynamics of the drug. ${ }^{5-7}$ ) The efficacy of various drugs varies according to the dosing time. ${ }^{8)}$ It is important to consider the chronopharmacological profiles of each drug in order to provide effective medication.

Depression is one of the diseases thought to be affected by the circadian system. Depressive symptoms relate to the chronotype ${ }^{9)}$ the symptoms of the depression show diurnal variations, ${ }^{10)}$ certain types of depression are developed according to the length of daylight, ${ }^{11)}$ and the modification of the circadian systems is sometimes effective for treating patients. ${ }^{12-14)}$ The monoamine hypothesis proposes that the reduction of monoamine systems in the brain causes depression. However, the interactions between the circadian system and various pathophysiologies are not yet thoroughly understood.

Many therapeutic drugs have been developed for depression, and various drugs such as tricyclic antidepressants, selective serotonin reuptake inhibitors (SSRIs), and serotoninnoradrenaline reuptake inhibitors (SNRIs) are currently used in clinical settings. Most of these drugs modify the monoaminergic systems in the brain to exert their antidepressant activity.

In rodent models used to assess the effectiveness of antidepressants, the forced swim test (FST) is one of the most frequently used behavioral tests. Since the FST has high predictive validity, reliability and robustness, this test is useful and widely used for screening and analysis of antidepressant activity. ${ }^{15)}$ The FST is usually conducted using normal rats or mice, and the reduction of the immobility time is used as an indicator of antidepressant activity. ${ }^{16)}$ The modified version of FST developed by Lucki and colleagues can not only detect the antidepressant effects of various drugs; it can also discriminate serotonergic and noradrenergic activities of the drugs. ${ }^{17)}$ This test is thus useful to evaluate antidepressant activity of various compounds and to analyze the activity's underlying mechanisms. ${ }^{18)}$

Several research groups have investigated the effects of dosing time on the activity of antidepressants in clinical settings and animal models. ${ }^{19)}$ It was proposed that in mice, the activity rhythm of serotonin transporter (SERT) in the midbrain plays a key role in the induction of the dosing time-dependent activity rhythm of the SSRI fluvoxamine. ${ }^{20)}$ However, there are few reports on the mechanism of the chronopharmacological activity of antidepressants, and the effects of other neural systems such as the noradrenergic system remain to be clarified. Since clinical studies have suggested that different drugs show different chronopharmacological profiles, ${ }^{21-24)}$ it is important to analyze various drugs with different mechanisms of action. In this study, we attempted to determine the effects of dosing time on the activity of the dual-action SNRI milnacipran with the use of the modified FST, and we analyzed the mechanism underlying milnacipran's chronopharmacological action.

\section{MATERIALS AND METHODS}

Chemicals Pentobarbital was obtained from Kyoritsu 
Seiyaku (Tokyo, Japan). Milnacipran hydrochloride and other chemicals were obtained from Wako Pure Chemical Industries, Ltd. (Osaka, Japan).

Animals Male adult Wistar-Hannover rats (200-250g) were obtained from CLEA Japan (Tokyo, Japan), and were maintained in an air-conditioned room at $24 \pm 2{ }^{\circ} \mathrm{C}$ with a 12/12-h light/dark cycle (lights on at 7:00 a.m.). The rats had free access to food and water. The time of the day is expressed as the zeitgeber time (ZT), defined as follows: the light onset time (7:00 a.m. clock time) is ZT0; the dark onset time (7:00 p.m. clock time) is ZT12, and ZT24 is the ZT0 of the next day.

Animal maintenance and treatments were in accordance with the general recommendations of animal protection legislation in Japan. All procedures were approved by the Institutional Animal Care and Use Committee of Josai International University.

Forced Swim Test We used a modified FST according to the method of Detke et al. ${ }^{25)}$ On the day before measurement, the rat was individually placed in a tank made of an opaque plastic cylinder (24-cm dia., $50-\mathrm{cm}$ height) filled with roomtemperature water $\left(24 \pm 2^{\circ} \mathrm{C}\right)$ to a depth of $30 \mathrm{~cm}$. The rat was left in the tank for $10 \mathrm{~min}$, then removed from the cylinder, allowed to dry in a clean cage, and returned to its home cage. On the next day of the first exposure, the rat was per-orally administered 40 or $60 \mathrm{mg} / \mathrm{kg}$ (as a hydrochloride salt) of milnacipran at 8:00a.m. (ZT1) or 8:00 p.m. (ZT13). Control rat was administered saline. One hour after the administration, the rat was again placed in the tank of room temperature water, and its behaviors were analyzed for $5 \mathrm{~min}$ : at regular 5-s intervals, a trained observer recorded which of the following three behaviors was predominant: immobility, swimming, or climbing. The total counts for each behavior over the $5 \mathrm{~min}$ test session were analyzed as the FST score.

Analysis of Plasma and Brain Contents of Milnacipran Rats were per-orally administered milnacipran at ZT1 or ZT13. One hour after the administration, they were anesthetized with pentobarbital, and the blood and whole brain samples were obtained. The blood sample was centrifuged at $1200 \times \boldsymbol{g}$ for $10 \mathrm{~min}$ to obtain plasma. The plasma and brain samples were immediately frozen in liquid nitrogen and kept at $-80^{\circ} \mathrm{C}$ until use.

(A)

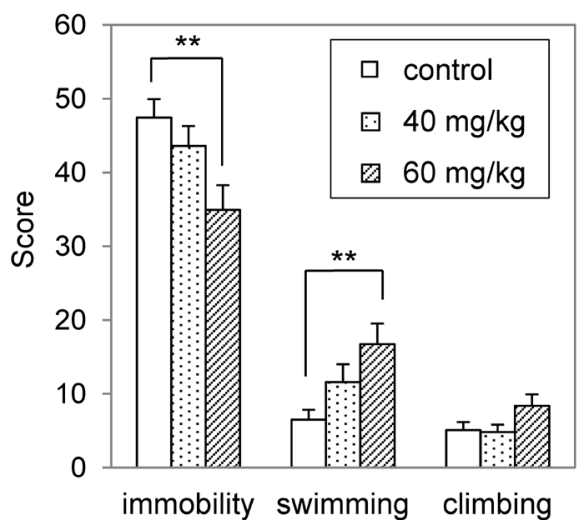

Venlafaxine at $10 \mathrm{nmol} / \mathrm{sample}$ was added to $1 \mathrm{~mL}$ of plasma as an internal standard. The samples were alkalinized with $1.5 \mathrm{~mL}$ of $1 \mathrm{M} \mathrm{NaOH}$ and extracted with $2 \mathrm{~mL}$ of dichloromethane/2-propanol $/ n$-hexane $(60 / 14 / 26)$. The extract was evaporated at $40^{\circ} \mathrm{C}$ under a nitrogen gas stream, reconstituted with $0.1 \mathrm{~mL}$ of $50 \mathrm{~mm}$ sodium phosphate buffer ( $\mathrm{pH} 3.8$ )/ acetonitrile $(80 / 20)$, and then filtered through a $0.45-\mu \mathrm{m}$ filter. A portion of the filtrate was subjected to an HPLC analysis as described below.

The brains were homogenized in a Polytron (Kinematica, Switzerland) with 5 volumes of $0.2 \mathrm{M}$ perchloric acid containing $\quad 0.1 \mathrm{~mm}$ ethylenediamine- $N, N, N^{\prime}, N^{\prime}$-tetraacetic acid (EDTA). Venlafaxine at $10 \mathrm{nmol} / \mathrm{sample}$ was added as an internal standard. The homogenate was centrifuged at $20000 \times \boldsymbol{g}$ for $15 \mathrm{~min}$ at $4^{\circ} \mathrm{C}$, and the supernatant was alkalinized with $0.5 \mathrm{~mL}$ of $8 \mathrm{M} \mathrm{NaOH}$. The sample was then extracted, evaporated, reconstituted, and filtered in the same way as the plasma samples.

Milnacipran was analyzed by HPLC-UV. An LC10AD/SPD-10A (Shimadzu, Kyoto, Japan) system was used, and the conditions were as follows: column, Gemini ODS ( $2.1 \mathrm{~mm}$ i.d. $\times 150 \mathrm{~mm}$, Phenomenex, Torrance, CA, U.S.A.) with a precolumn (SecurityGuard, Phenomenex); mobile phase, $80 \% 50 \mathrm{~mm}$ sodium phosphate buffer ( $\mathrm{pH} 3.8$ ), 20\% acetonitrile; flow, $0.2 \mathrm{~mL} / \mathrm{min}$; column temperature, $40^{\circ} \mathrm{C}$; detection, $200 \mathrm{~nm}$.

Analysis of Monoamine Neurotransmitters Brain samples were collected at ZT2, 6, 10, 14, 18, and 22. Rats were anesthetized with pentobarbital and sacrificed by decapitation. The brains were rapidly dissected out and divided into sections. The prefrontal cortex (PFC), hippocampus, and brain stem were immediately frozen in liquid nitrogen and kept at $-80^{\circ} \mathrm{C}$ until use.

The divided brain tissues were homogenized in a Polytron with 5 volumes of $0.2 \mathrm{M}$ perchloric acid containing $0.1 \mathrm{~mm}$ EDTA. Isoproterenol at $1 \mathrm{nmol} / \mathrm{sample}$ was added as an internal standard. The homogenate was centrifuged at $20000 \times \boldsymbol{g}$ for $15 \mathrm{~min}$ at $4^{\circ} \mathrm{C}$, and the supernatant was adjusted to $\mathrm{pH} 3.0$ with $1 \mathrm{M}$ sodium acetate, and then filtered through a $0.45-\mu \mathrm{m}$ filter. A portion of the filtrate was subjected to an HPLC analysis as described below.

Monoamine neurotransmitters and their metabolites were

(B)

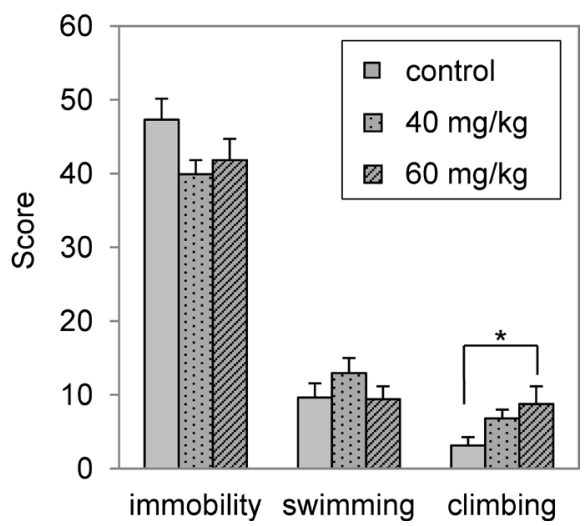

Fig. 1. Dosing Time-Dependent Activity of Milnacipran in the FST

The scores for immobility, swimming, and climbing in the morning (ZT1) (A) and in the evening (ZT13) (B) are shown. Each column represents mean+ S.E.M. of 11 to 12 rats. $* p<0.05, * * p<0.01$ by Dunnett's test. 
analyzed with HPLC-electrochemical detection (ECD). Serotonin (5-hydroxytryptamine, 5HT), 5-hydroxyindolacetic acid (5HIAA), noradrenaline (NA), and 3-methyoxy-4-hydroxyphenylethylene glycol (MHPG) were analyzed. An LC-10AD (Shimadzu, Kyoto, Japan)/Eicom ECD-700 (Eicom, Kyoto, Japan) system was used, and the conditions were as follows: column, SC-5ODS (3.0 mm i.d. $\times 150 \mathrm{~mm}$, Amuza, San Diego, CA, U.S.A.) with a precolumn (PREPAK-AC, Amuza); mobile phase, $87 \% 0.1 \mathrm{M}$ sodium acetate/0.1 M citric acid buffer $(\mathrm{pH}$
(A)

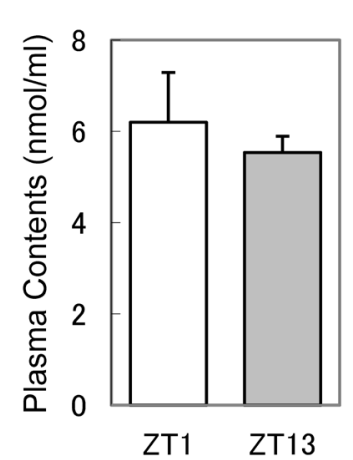

(B)

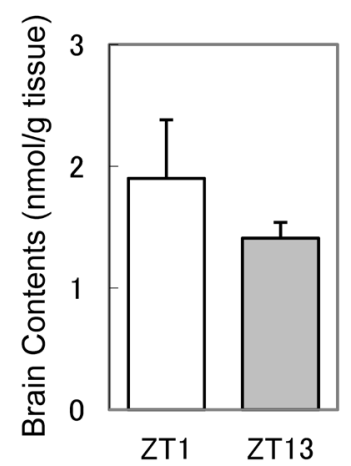

Fig. 2. Influence of Dosing Time on the Levels of Milnacipran in the Plasma (A) and in the Brain (B)

Milnacipran was administered at ZT1 or ZT13, and the levels were measured $1 \mathrm{~h}$ later. Each column represents mean+ S.E.M. of 4 rats. No significant difference was detected between ZT1 and ZT13.
3.5), $13 \%$ methanol, containing $5 \mathrm{mg} / \mathrm{L}$ EDTA and $190 \mathrm{mg} / \mathrm{L}$ sodium octanesulfonate; flow, $0.5 \mathrm{~mL} / \mathrm{min}$; electrode, Amuza WE-3G graphite; reference, Amuza RE-100 Ag-AgCl; applied voltage, $700 \mathrm{mV}$ vs. $\mathrm{Ag}-\mathrm{AgCl}$.

Statistical Analysis Data are expressed as the mean \pm standard error of the mean (S.E.M.). In the behavioral analysis, statistically significant differences between the experimental and control groups were analyzed by Dunnett's test (Fig. 1). Comparisons of two groups were analyzed by Student's $t$-test (Fig. 2). Intra-day fluctuations of the obtained data (Fig. 3) were analyzed by one-way ANOVA. Pairwise multiple comparisons were analyzed by Tukey's test (Fig. 3). The significance level was set at $p<0.05$.

All statistical analyses were performed with EZR (Saitama Medical Center, Jichi Medical University, Saitama, Japan), which is a graphical user interface for R (The R Foundation for Statistical Computing, Vienna, Austria). More precisely, EZR is a modified version of $\mathrm{R}$ commander designed to add statistical functions frequently used in biostatistics. ${ }^{26)}$

\section{RESULTS}

The Dosing Time-Dependent Antidepressant Activity of Milnacipran Milnacipran ( 40 or $60 \mathrm{mg} / \mathrm{kg}$ as a hydrochloride salt) was administered to rats in the morning (ZT1) or evening (ZT13), and the FST was conducted $1 \mathrm{~h}$ later. The results at ZT1 and ZT13 are shown in Fig. 1. The morning group treated with milnacipran showed a dose-dependent
(A)

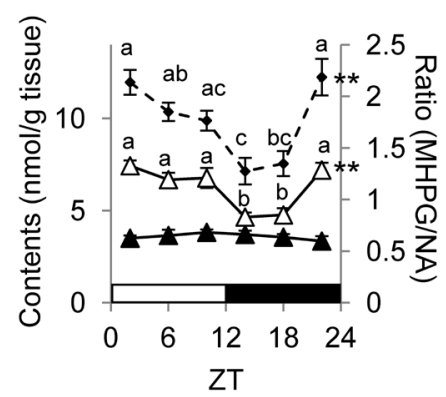

(D)

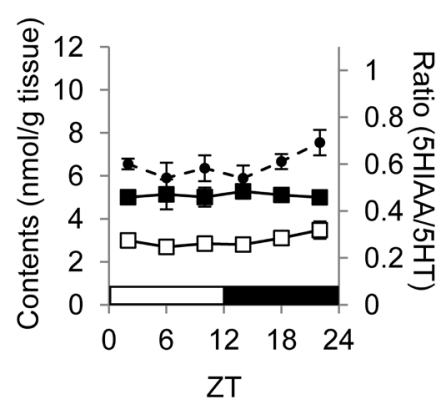

(B)

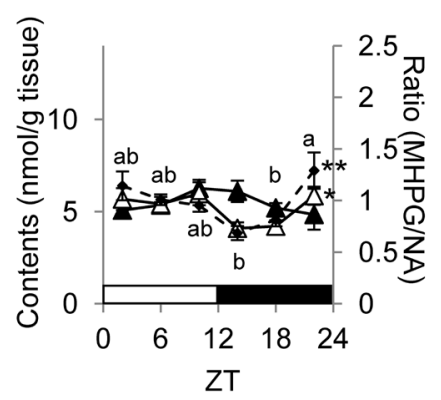

(E)

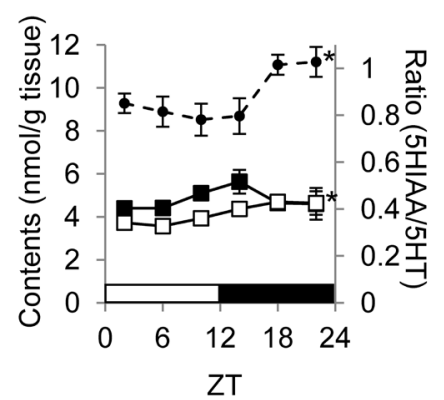

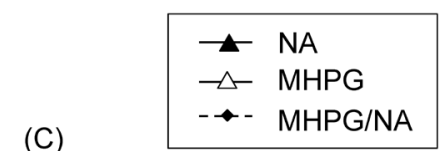

(C)
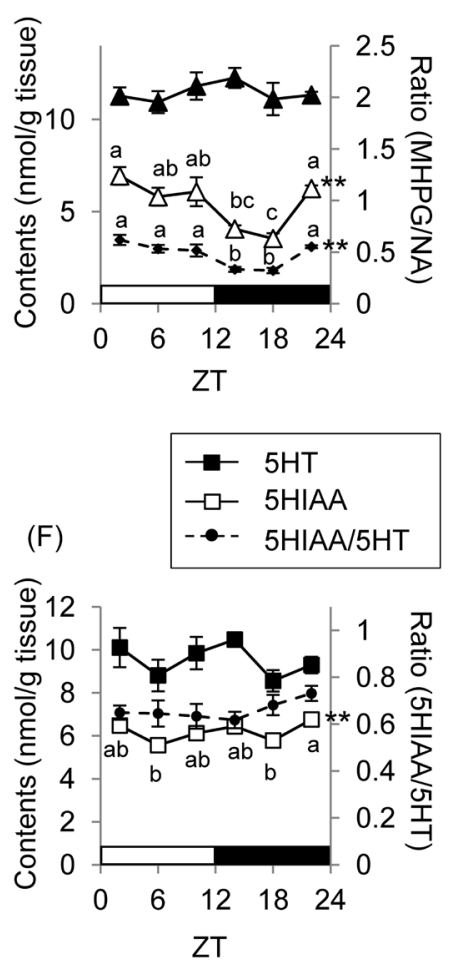

Fig. 3. Intra-day Fluctuations of 5HT, NA, Their Metabolites and Turnover Rate in Rat Brain

The levels of each compound and the turnover rate (5HIAA/5HT and MHPG/NA) in the PFC (A, D), hippocampus (B, E), and brain stem (C, F) were measured at ZT2, $6,10,14,18$, and 22 . The open and closed bars on the horizontal axes represent the light and dark periods of the day, respectively. Each point represents mean \pm S.E.M. of 4 rats. ${ }^{*} p<0.05, * * p<0.01$ by one-way ANOVA. Different letters $(\mathrm{a}, \mathrm{b}, \mathrm{c})$ on each line indicate $p<0.05$ between respective time points by Tukey's test. 
reduction of immobility and increased swimming $(p<0.01$ $v s$. control, respectively). The evening group showed a dosedependent increase in climbing $(p<0.05$ vs. control), but did not show a significant reduction of immobility at this dose.

The Influence of Dosing Time on the Plasma and Brain Levels of Milnacipran The plasma and brain of the rats were collected $1 \mathrm{~h}$ after the administration of $60 \mathrm{mg} / \mathrm{kg}$ of milnacipran hydrochloride, and the plasma and brain levels of milnacipran are shown in Fig. 2. There was no significant difference in these levels between ZT1 and ZT13 ( $p=0.58$ and 0.36 , respectively).

Circadian Fluctuation of the Serotonergic and Noradrenergic Systems The levels of $5 \mathrm{HT}$, NA, and their metabolites (5HIAA and MHPG) were measured in the PFC, hippocampus, and brain stem. The intra-day fluctuations of these compounds and the turnover of each neurotransmitter (5HIAA/5HT and MHPG/NA) are shown in Fig. 3. Although 5HT and NA did not show large fluctuations, the ANOVA revealed significant fluctuations of 5HIAA, 5HIAA/5HT, MHPG, and MHPG/NA in some regions (5HIAA in hippocampus: $F=3.31, p=0.03$; 5HIAA in brain stem: $F=4.50$, $p<0.01$; 5HIAA/5HT in hippocampus: $F=3.33, p=0.03$; MHPG in PFC: $F=10.27, p<0.01$; MHPG in hippocampus: $F=3.13, \quad p=0.03$; MHPG in brain stem: $F=8.71, p<0.01$; MHPG/NA in PFC: $F=9.34, p<0.01 ;$ MHPG/NA in hippocampus: $F=4.38, p<0.01$; MHPG/NA in brain stem: $F=10.48$, $p<0.01)$. Tukey's test detected the statistically significant difference between time points in 5HIAA in brain stem (ZT6 vs. ZT22: $p=0.01, \mathrm{ZT} 18$ vs. ZT22: $p=0.046)$; MHPG in PFC (ZT2 vs. ZT14: $p<0.01$, ZT2 vs. ZT18: $p<0.01$, ZT6 vs. ZT14: $p=0.02$, ZT6 vs. ZT18: $p=0.02$, ZT14 vs. ZT22: $p<0.01$, ZT18 vs. ZT22: $p<0.01$ ) and brain stem (ZT2 vs. ZT14: $p<0.01$, ZT2 vs. ZT18: $p<0.01$, ZT6 vs. ZT18: $p=0.02$, ZT10 vs. ZT18: $p=0.01$, ZT14 vs. ZT22: $p=0.03$, ZT18 vs. ZT22: $p<0.01)$; and MHPG/NA in PFC (ZT2 vs. ZT14: $p<0.01$, ZT2 vs. ZT18: $p<0.01$, ZT6 vs. ZT14: $p=0.04$, ZT14 vs. ZT22: $p<0.01$, ZT18 vs. ZT22: $p<0.01$ ), hippocampus (ZT14 vs. ZT22: $p<0.01$, ZT18 vs. ZT22: $p=0.04)$, and brain stem (ZT2 vs. ZT14: $p<0.01$, ZT2 vs. ZT18: $p<0.01$, ZT6 vs. ZT14: $p=0.02$, ZT6 vs. ZT18: $p=0.01$, ZT10 vs. ZT14: $p=0.03$, ZT10 vs. ZT18: $p=0.02$, ZT14 vs. ZT22: $p<0.01$, ZT18 vs. ZT22: $p<0.01$ ).

\section{DISCUSSION}

This is the first study to reveal the chronopharmacological profile of the antidepressant activity of milnacipran in rats. Milnacipran showed dosing time-dependent activity in the FST (Fig. 1). A large reduction of immobility by milnacipran was observed in the morning (ZT1) compared to the evening (ZT13), suggesting that milnacipran exerts its antidepressant activity more effectively in the morning. We also observed that the rats' swimming behavior was increased by milnacipran in the morning, and the climbing behavior was increased by milnacipran in the evening. The different responses to milnacipran in FST behaviors between the morning and evening suggest that the mechanism of action responsible for the antidepressant activity of milnacipran could differ according to the time of day.

In the FST, increased swimming and climbing reflect the activation of the serotonergic and noradrenergic systems, respectively. ${ }^{17,25)}$ SSRIs such as fluoxetine reduced immobility, increased swimming, but did not affect climbing. ${ }^{27,28)}$ Noradrenaline-specific reuptake inhibitors such as reboxetine reduced immobility and increased climbing. ${ }^{29)}$ As shown in Fig. 1 , the rats' increase in swimming behavior was greater at ZT1, whereas their increase in climbing was greater at ZT13. These results suggest that milnacipran exerts its antidepressant activity by activating the serotonergic system in the morning and activating the noradrenergic system in the evening.

Rénéric and Lucki assessed the behavioral pattern of milnacipran-treated rats in the FST, and they reported that milnacipran produced a reduction of immobility and an increase in climbing. ${ }^{30)}$ This behavioral pattern resembles our results at ZT13, although the responses in their study were larger than the present study. The larger responses may have occurred because Rénéric and Lucki administered milnacipran three times before the FST. That administration procedure is often used, and is known to enhance the response in the FST. ${ }^{31)}$ Since they conducted the FST in the latter half of the subjective day (lights-on duration), their results are consistent with ours. The serotonergic system might be more sensitive to milnacipran than the noradrenergic system in the morning. The relative sensitivity of the noradrenergic system to milnacipran might increase according to time, and the noradrenergic effects might be dominant in the afternoon and in the evening.

Ushijima et al. reported that the anti-immobility effects of amitriptyline and fluvoxamine were potent in the early dark period. ${ }^{20)}$ Our present findings are inconsistent with their results. This is likely to be due to several differences between their study and the present investigation. First, Ushijima et al. used mice and we used rats. Although both mice and rats are nocturnal, several chronobiological characters such as the period length of the biological clock differ between mice and rats. This might contribute to the difference in chronopharmacological profiles. Second, the mechanisms of action of the drugs are different. Milnacipran is an SNRI, and fluvoxamine is an SSRI. Activation of the noradrenergic system by the inhibition of noradrenaline transporter (NAT) might contribute to the difference between milnacipran and fluvoxamine. Amitriptyline inhibits NAT, SERT, and several serotonergic and adrenergic receptors, especially $5 \mathrm{HT}_{2}$ receptor and $\alpha_{1}$ adrenergic receptor. ${ }^{32)}$ Since the activation of $\alpha_{1}$-adrenergic receptors leads to antidepressant effects, ${ }^{33)}$ the inhibition of $\alpha_{1}$-adrenergic receptors may reduce the antidepressant effect of amitriptyline via the noradrenergic system. This effect might contribute to the difference between milnacipran and amitriptyline.

Chronopharmacological effects could be induced by pharmacokinetic effects and/or pharmacodynamic effects. We first investigated the pharmacokinetic effects on the dosing time-dependent action of milnacipran. Desmethylation by CYP3A4 and glucuronidation are major metabolic pathways of milnacipran. ${ }^{34)}$ Since none of the known metabolites of milnacipran show antidepressant activity, the circadian rhythm of its metabolization could induce the dosing time-dependent activity of milnacipran. As shown in Fig. 2, however, the levels of milnacipran at $1 \mathrm{~h}$ after the administration, when the FST was carried out, were not different between ZT1 and ZT13. A previous study indicated that the brain contents of a drug at the time point of a behavioral analysis would be crucial for the chronopharmacological analysis of antidepressants. ${ }^{20)}$ Although we could not determine the pharmacokinetic param- 
eters such as the $C_{\max }$ and $T_{1 / 2}$ of milnacipran in the present study, we speculate that the pharmacokinetic effect was not sufficient to induce the chronopharmacological action of milnacipran in the FST.

We next investigated the pharmacodynamic effects. Milnacipran activates the serotonergic and noradrenergic systems by inhibiting transporters that take up serotonin or noradrenaline from the synaptic clefts in the brain. A circadian fluctuation of the activity of these systems could induce the dosing time-dependent action of milnacipran. Various studies have analyzed the circadian rhythms of the monoamine system in the brain, and they showed that the circadian profiles of the monoamine system vary among different species and strains. ${ }^{19,35,36)}$ In the Wistar-Hannover rats used in the present study, intra-day fluctuations were detected in the serotonergic and noradrenergic systems (Fig. 3). The turnover rate (5HIAA/5HT and MHPG/NA) showed significant fluctuations in various regions. In the hippocampus, 5HIAA/5HT showed a peak during ZT18-22 and a nadir during ZT10-14, whereas MHPG/NA showed a peak during ZT22-2 and a nadir during ZT14-18. The turnover rates are indicators of the activation of the respective neuronal system. This difference between the activity rhythms of the serotonergic and noradrenergic systems might have contributed to the dosing time-dependent profiles of milnacipran in the FST.

The $K_{\mathrm{i}}$ values of milnacipran for SERT and NAT were reported as 8.5 and $31 \mathrm{~nm}$, respectively. ${ }^{37)}$ Since the brain contents of milnacipran shown in Fig. 2 seemed sufficient to inhibit SERT and NAT, the pharmacological effect of milnacipran might depend on the basal activity of 5HT and NA neurons. Larger amounts of neurotransmitters are released at the peak time of the neuronal activity. These neurotransmitters are accumulated in the synaptic cleft by the action of milnacipran, and they stimulate postsynaptic neurons efficiently, which results in the antidepressant activity. The low neuronal activity of the 5HT and NA system during the early dark period (Fig. 3) might cause the low antidepressant activity at ZT13 in the FST. The relatively high level of 5HT neuronal activity in the morning might have induced the rats' swimming in the FST, and the reduction of 5HT neural activity in the evening might have changed the balance of NA and 5HT, which led to the increase in climbing in the FST.

We consider that it is difficult to explain the chronopharmacological action of milnacipran completely by the activity rhythms of monoamine neurotransmitters. The difference of the serotonergic activity between ZT2 and ZT14 was small. The noradrenergic activity was reduced in the evening, while the FST suggested that the noradrenergic action was potent in the evening. These inconsistencies between the pharmacological activity and the biochemical activity suggest that the other mechanisms should affect the action of milnacipran. We suspect that the circadian fluctuation of other factors in NA and 5HT system such as receptors and transporters also influence the action of milnacipran. The circadian rhythm of these factors and neurotransmitters might cooperatively determine the chronopharamacological profile of milnacipran.

We analyzed the rhythms of the serotonergic and noradrenergic systems in the rat $\mathrm{PFC}$, hippocampus, and brain stem, because these regions are known to be important for depression, antidepressant activity, and monoamine neurotransmission. Both the serotonergic and noradrenergic systems originate from nuclei in the brain stem, and modulation in this region affects various brain functions. ${ }^{38,39)}$ Many studies have indicated the importance of the PFC and hippocampus in the pathogenesis of depression and the activity of antidepressants. For example, the reduction of glucose metabolism and insufficiency of neural activity were observed in the PFC of depressed patients. ${ }^{40-42)}$ Chronic imipramine treatment increased the expression of the plasticity-associated proteins in the PFC and hippocampus. ${ }^{43}$ Several antidepressants increased the neurogenesis of hippocampus, which was critical to the antidepressants' effects. ${ }^{44,45)}$

In our comparison of the three regions in the present study, the rhythms of both the serotonergic and noradrenergic systems were relatively potent in the PFC and hippocampus, and weak in the brain stem. We suspect that the contributions of the PFC and hippocampus to the chronopharmacological activity of the antidepressant were greater than that of the brain stem. However, the amplitude of the rhythm observed in this study was at most $30 \%$ of the mean level, which might be too small to explain the effects observed in the FST. Although the activity rhythm of the monoamine system might contribute to some extent, other mechanisms should also be expected to affect the chronopharmacological effects of antidepressants.

Several studies have reported the circadian fluctuation of some receptors in monoamine systems. The pharmacological response of $5 \mathrm{HT}_{1 \mathrm{~A}}$ agonist was high at mid-night and low at mid-day, ${ }^{46)}$ whereas that of $5 \mathrm{HT}_{2 \mathrm{~A} / 2 \mathrm{C}}$ agonist was high in a late light period and low in a late dark period. ${ }^{47,48)}$ Ushijima et al. showed that the activity of SERT was higher in an early dark period than in an early light period in mice, and they reported that this effect caused the dosing time-dependent pharmacological action of SSRIs. ${ }^{20)}$ The circadian activity rhythm of receptors and transporters in monoamine systems might contribute to the chronopharmacological effects of milnacipran and other antidepressants. The circadian profiles of SERT, NAT and other receptors should be further studied.

In this study, antidepressant activity was evaluated by the FST. We used normal naïve rats and administered milnacipran only once in the FST. These conditions were different from clinical cases. Since the FST with normal rodents has high predictive validity, ${ }^{15)}$ the results in Fig. 1 suggest the possibility that the clinical efficacy of milnacipran could be influenced by dosing time. The analysis of the effect of chronic antidepressant treatment, the analysis using animal models of depression, and clinical trial with depressive patients could further clarify the importance of the chronopharmacological action of antidepressant.

Our present findings revealed that milnacipran led to FST behaviors that differed between the morning and evening, suggesting that the serotonergic and the noradrenergic systems were activated differently according to the dosing time. This suggests that antidepressants with different mechanisms of action might show different circadian profiles, and the best timing of administration might be different for each drug. Knowledge of the optimal administration times is important for optimizing the dosing times in drug therapy. Although the precise mechanisms should be further analyzed, the differences in circadian profiles between 5HT and NA could play an important role in the chronopharmacological effects of antidepressants. 
Acknowledgment We are grateful for the assistance given by N. Kodaira, A. Suzuki, and C. Tanaka.

Conflict of Interest The authors declare no conflict of interest.

\section{REFERENCES}

1) Hastings MH, Reddy AB, Maywood ES. A clockwork web: circadian timing in brain and periphery, in health and disease. Nat. Rev. Neurosci., 4, 649-661 (2003).

2) Savvidis C, Koutsilieris M. Circadian rhythm disruption in cancer biology. Mol. Med., 18, 1249-1260 (2012).

3) Takeda N, Maemura K. Circadian clock and cardiovascular disease. J. Cardiol., 57, 249-256 (2011).

4) Wulff K, Gatti S, Wettstein JG, Foster RG. Sleep and circadian rhythm disruption in psychiatric and neurodegenerative disease. Nat. Rev. Neurosci., 11, 589-599 (2010).

5) Levi F, Schibler U. Circadian rhythms: mechanisms and therapeutic implications. Annu. Rev. Pharmacol. Toxicol., 47, 593-628 (2007).

6) Ohdo S, Koyanagi S, Matsunaga N, Hamdan A. Molecular basis of chronopharmaceutics. J. Pharm. Sci., 100, 3560-3576 (2011).

7) Dallmann R, Brown SA, Gachon F. Chronopharmacology: new insights and therapeutic implications. Annu. Rev. Pharmacol. Toxicol., 54, 339-361 (2014).

8) Ohdo S. Chronotherapeutic strategy: rhythm monitoring, manipulation and disruption. Adv. Drug Deliv. Rev., 62, 859-875 (2010).

9) $\mathrm{Au} \mathrm{J}$, Reece J. The relationship between chronotype and depressive symptoms: a meta-analysis. J. Affect. Disord., 218, 93-104 (2017).

10) Moffoot APR, O'Carroll RE, Bennie J, Carroll S, Dick H, Ebmeier KP, Goodwin GM. Diurnal variation of mood and neuropsychological function in major depression with melancholia. J. Affect. Disord., 32, 257-269 (1994)

11) Lewy AJ, Lefler BJ, Emens JS, Bauer VK. The circadian basis of winter depression. Proc. Natl. Acad. Sci. U.S.A., 103, 7414-7419 (2006).

12) Golden RN, Gaynes BN, Ekstrom RD, Hamer RM, Jacobsen FM, Suppes T, Wisner KL, Nemeroff CB. The efficacy of light therapy in the treatment of mood disorders: a review and meta-analysis of the evidence. Am. J. Psychiatry, 162, 656-662 (2005).

13) Bechtel W. Circadian rhythms and mood disorders: are the phenomena and mechanisms causally related? Front. Psychiatry, 6, 118 (2015).

14) Kronfeld-Schor N, Einat H. Circadian rhythms and depression: human psychopathology and animal models. Neuropharmacology, 62, 101-114 (2012).

15) Slattery DA, Cryan JF. Using the rat forced swim test to assess antidepressant-like activity in rodents. Nat. Protoc., 7, 1009-1014 (2012).

16) Porsolt RD, Le Pichon M, Jalfre M. Depression: a new animal model sensitive to antidepressant treatments. Nature, 266, 730-732 (1977).

17) Cryan JF, Markou A, Lucki I. Assessing antidepressant activity in rodents: recent developments and future needs. Trends Pharmacol. Sci., 23, 238-245 (2002).

18) Lim DW, Jung J-W, Park J-H, Baek N-I, Kim YT, Kim I-H, Han D. Antidepressant-like effects of Sanggenon $\mathrm{G}$, isolated from the root bark of Morus alba, in rats: involvement of the serotonergic system. Biol. Pharm. Bull., 38, 1772-1778 (2015).

19) Nagayama H. Influences of biological rhythms on the effects of psychotropic drugs. Psychosom. Med., 61, 618-629 (1999).

20) Ushijima K, Sakaguchi H, Sato Y, To H, Koyanagi S, Higuchi S, Ohdo S. Chronopharmacological study of antidepressants in forced swimming test of mice. J. Pharmacol. Exp. Ther., 315, 764-770 (2005).

21) Nakano S, Hollister LE. Chronopharmacology of amitriptyline.
Clin. Pharmacol. Ther., 33, 453-459 (1983).

22) Nagayama $H$, Nagano K, Ikezaki A, Tashiro T. Double-blind study of the chronopharmacotherapy of depression. Chronobiol. Int., $\mathbf{8}$, 203-209 (1991).

23) Srinivas NR, Shyu WC, Gleason C, Schuster A, Greene DS, Barbhaiya RH. Effects of age, gender, and diurnal variation on the steady-state pharmacokinetics of BMS-181101, an antidepressant, in healthy subjects. Clin. Pharmacol. Ther., 62, 408-416 (1997).

24) Bougerolle AM, Chabard JL, Jbilou M, Dordain G, Eschalier A, Aumaitre O, Gaillot J, Piron JJ, Petit J, Berger JA. Chronopharmacokinetic and bioequivalence studies of two formulations of trimipramine after oral administration in man. Eur. J. Drug Metab. Pharmacokinet., 14, 139-144 (1989).

25) Detke MJ, Rickels M, Lucki I. Active behaviors in the rat forced swimming test differentially produced by serotonergic and noradrenergic antidepressants. Psychopharmacology, 121, 66-72 (1995).

26) Kanda Y. Investigation of the freely available easy-to-use software 'EZR' for medical statistics. Bone Marrow Transplant., 48, 452-458 (2013).

27) $\overline{\text { Detke }}$ MJ, Johnson J, Lucki I. Acute and chronic antidepressan drug treatment in the rat forced swimming test model of depression. Exp. Clin. Psychopharmacol., 5, 107-112 (1997).

28) Page ME, Detke MJ, Dalvi A, Kirby LG, Lucki I. Serotonergic mediation of the effects of fluoxetine, but not desipramine, in the rat forced swimming test. Psychopharmacology, 147, 162-167 (1999).

29) Cryan JF, Markou A, Lucki I. Assessing antidepressant activity in rodents: Recent developments and future needs. Trends Pharmacol. Sci., 23, 238-245 (2002).

30) Rénéric JP, Lucki I. Antidepressant behavioral effects by dual inhibition of monoamine reuptake in the rat forced swimming test. Psychopharmacology, 136, 190-197 (1998).

31) Borsini F, Meli A. Is the forced swimming test a suitable model for revealing antidepressant activity? Psychopharmacology, 94, 147-160 (1988)

32) Richelson E, Nelson A. Antagonism by antidepressants of neurotransmitter receptors of normal human brain in vitro. J. Pharmacol. Exp. Ther., 230, 94-102 (1984).

33) Doze VA, Handel EM, Jensen KA, Darsie B, Luger EJ, Haselton JR, Talbot JN, Rorabaugh BR. $\alpha_{1 \mathrm{~A}^{-}}$and $\alpha_{1 \mathrm{~B}}$-adrenergic receptors differentially modulate antidepressant-like behavior in the mouse. Brain Res., 1285, 148-157 (2009).

34) Caccia S. Metabolism of the newer antidepressants. An overview of the pharmacological and pharmacokinetic implications. Clin. Pharmacokinet., 34, 281-302 (1998).

35) Quay WB. Regional and circadian differences in cerebral cortical serotonin concentrations. Life Sci., 4, 379-384 (1965).

36) Bhaskaran D, Radha E. Circadian variations in the monoamine levels and monoamine oxidase activity in different regions of the rat brain as a function of age. Exp. Gerontol., 19, 153-170 (1984).

37) Mochizuki D, Tsujita R, Yamada S, Kawasaki K, Otsuka Y, Hashimoto S, Hattori T, Kitamura Y, Miki N. Neurochemical and behavioural characterization of milnacipran, a serotonin and noradrenaline reuptake inhibitor in rats. Psychopharmacology, 162, 323-332 (2002).

38) Jacobs BL, Azmitia EC. Structure and function of the brain serotonin system. Physiol. Rev., 72, 165-229 (1992).

39) Foote SL, Bloom FE, Aston-Jones G. Nucleus locus ceruleus: New evidence of anatomical and physiological specificity. Physiol. Rev., 63, 844-914 (1983)

40) Baxter LR Jr, Schwartz JM, Phelps ME, Mazziotta JC, Guze BH, Selin CE, Gerner RH, Sumida RM. Reduction of prefrontal cortex glucose metabolism common to three types of depression. Arch. Gen. Psychiatry, 46, 243-250 (1989).

41) Harvey P-O, Fossati P, Pochon J-B, Levy R, LeBastard G, Lehéricy S, Allilaire J-F, Dubois B. Cognitive control and brain resources in major depression: An fMRI study using the $n$-back task. Neuroim- 
age, 26, 860-869 (2005)

42) Wagner G, Sinsel E, Sobanski T, Köhler S, Marinou V, Mentzel H-J, Sauer H, Schlösser RGM. Cortical inefficiency in patients with unipolar depression: An event-related fMRI study with the Stroop Task. Biol. Psychiatry, 59, 958-965 (2006).

43) Sairanen M, O’Leary OF, Knuuttila JE, Castrén E. Chronic antidepressant treatment selectively increases expression of plasticityrelated proteins in the hippocampus and medial prefrontal cortex of the rat. Neuroscience, 144, 368-374 (2007).

44) Malberg JE, Eisch AJ, Nestler EJ, Duman RS. Chronic antidepressant treatment increases neurogenesis in adult rat hippocampus. $J$. Neurosci., 20, 9104-9110 (2000).

45) Santarelli L, Saxe M, Gross C, Surget A, Battaglia F, Dulawa S,
Weisstaub N, Lee J, Duman R, Arancio O, Belzung C, Hen R. Requirement of Hippocampal neurogenesis for the behavioral effects of antidepressants. Science, 301, 805-809 (2003).

46) Lu JQ, Nagayama H. Circadian rhythm in the response of central 5-HT1A receptors to 8-OH-DPAT in rats. Psychopharmacology, 123, 42-45 (1996).

47) Moser PC, Redfern PH. Circadian variation in behavioural responses to central 5-HT receptor stimulation in the mouse. Psychopharmacology, 86, 223-227 (1985).

48) Nagayama H, Lu J-Q. Circadian rhythm in the responsiveness of central 5-HT2A receptor to DOI in rats. Psychopharmacology, 127, $113-116$ (1996). 